\section{COVID-19: nueva escala para medir su relación estrecha con la ansiedad}

\section{COVID-19: new scale to measure its close relationship with anxiety}

\author{
Sakura Basaldua-Cabezudo,* \\ José Córdova-Tello* \\ Andrea Cadillo-Poma*
}

*Universidad Privada San Juan Bautista. Ica-Perú.

Citar como: Basaldua-Cabezudo S, Córdova-Tello J, Cadillo-Poma A. COVID-19: nueva escala para medir su relación estrecha con la ansiedad. Neumol Cir Torax. 2021; 80 (4): 321. https://dx.doi. org/10.35366/103461

\section{Sr. Editor:}

Hemos leído con atención el artículo Ansiedad en familiares de pacientes con COVID-19 hospitalizados en el Instituto Nacional de Enfermedades Respiratorias. ${ }^{1}$ El autor resalta la importancia de evaluar y cuantificar los síntomas de ansiedad en los familiares de pacientes con COVID-19 hospitalizados. Consideramos que es un tema de suma importancia, por lo que debería tener mayor énfasis durante este período de COVID-19 con la finalidad de evaluar las repercusiones en la salud mental.

A principios de 2020, la enfermedad por el nuevo coronavirus (COVID-19) comenzó a extenderse por toda China. Este rápido aumento de confirmaciones de casos y muertes ha ocasionado problemas como estrés, ansiedad y depresión tanto en el personal médico como en la población general. ${ }^{2}$ Este incremento de los casos confirmados y los decesos se extendió por todo el mundo, en el que Latinoamérica no fue excluida, además dichos problemas llegaron a convertirse en focos importantes de la pandemia.

En esta situación insólita es difícil predecir con exactitud y por tanto, poder estimar las consecuencias psicológicas y emocionales del COVID-19. Las investigaciones que llegan de China, primer país afectado, apuntan a que el miedo a lo desconocido y la incertidumbre pueden llegar a evolu- cionar a enfermedades mentales como los trastornos de estrés, ansiedad, depresión, somatización y conductas como aumento de consumo de alcohol y tabaco. ${ }^{3}$

El estudio Escala de ansiedad por coronavirus: un nuevo instrumento para medir síntomas de ansiedad asociados al COVID-19 nos plantea una nueva manera de medir los síntomas de ansiedad asociados específicamente a la pandemia de COVID-19, la cual consiste en una serie de cinco preguntas donde los participantes pueden obtener puntajes desde $0=$ nada y $4=$ todos los días dentro del período de las últimas dos semanas. ${ }^{4}$

Siendo el ítem 1 el que nos va ayudar a examinar si el paciente ha presentado mareos al momento de exponerse a información de COVID-19. Considerando de esta manera los mareos como uno de los síntomas característicos de un ataque de pánico y de un trastorno de ansiedad generalizada.

El ítem 2 nos ayuda a valorar si el paciente presenta alteraciones de sueño provocadas por una exposición a la información con relación al COVID-19, siendo éste un síntoma que puede ser causa o consecuencia de los trastornos de ansiedad.

Por otro lado, el ítem 3 examina los síntomas de inmovilidad tónica (como la incapacidad para moverse o gesticular algún sonido), síntoma presente en una respuesta fisiológica defensiva en función de una cercanía al peligro o miedo. La pérdida de apetito es evaluada por el ítem 4, que es muy característica en la depresión o en los ataques de pánico, siendo también una respuesta que tiene el organismo al miedo o pánico.

Por último, el ítem 5 evalúa la presencia de náuseas o problemas estomacales que tienen relación con la exposición a información de COVID-19, síntomas también relacionados como una respuesta al miedo o pánico. ${ }^{4}$

El estudio dio como resultado que esta escala de ansiedad por coronavirus (CAS, por sus siglas en inglés) no sólo nos ayuda a diferenciar entre personas que presentan o no ansiedad disfuncional, sino también nos demostró que existe una asociación al diagnóstico positivo de COVID-19, a un consumo excesivo de alcohol o drogas, a una desesperanza extrema y además, al pensamiento suicida. ${ }^{4}$

Los instrumentos de evaluación tradicionales como el Patient Health Ques- tionnaire (PHQ-9) o Generalized Anxiety Disorder (GAD-7) podrían generar diagnósticos insuficientes o excesivos al no estar dirigidos a la identificación de síntomas relevantes y específicos de los problemas de salud mental asociados al COVID-19). ${ }^{5}$ Por esta razón se diseñó el instrumento EAC, que permite identificar problemas de salud mental relacionados con el COVID-19.

\section{CONCLUSIÓN}

La preocupación por infectarse y las medidas de confinamiento que nos hemos visto obligados a acatar por la pandemia de COVID-19, han generado en la población un incremento de ansiedad, depresión y miedo causando repercusiones en la salud mental. Por esta razón, la escala de ansiedad por coronavirus (EAC) presenta óptimas propiedades psicométricas para la investigación de los síntomas de ansiedad en relación al COVID-19 en la población en general.

\section{REFERENCIAS}

1. Ruiz-Aguilar CA, Urdapilleta-Herrera EDC, LaraRivas G. Ansiedad en familiares de pacientes con COVID-19 hospitalizados en el Instituto Nacional de Enfermedades Respiratorias. Neumol Cir Torax. 2021:80(2):100-104.

2. Liu S, Yang L, Zhang C, Xiang YT, Liu Z, Hu $S$, et al. Servicios de salud mental en línea en China durante el brote de COVID-19. The Lancet Psychiatry. 2020;7(4):e17.

3. Shigemura J, Ursano RJ, Morganstein JC, Kurosawa M, Benedek DM. Respuestas públicas al nuevo coronavirus de 2019 (2019 $\square$ nCoV) en Japón: consecuencias para la salud mental y poblaciones objetivo. Psychiatry Clin Neurosci. 2020;74(4):281-282.

4. González-Rivera JA, Rosario-Rodríguez A, Cruz-Santos A, González-Rivera JA, RosarioRodríguez A, Cruz-Santos A. Escala de ansiedad por coronavirus: un nuevo instrumento para medir síntomas de ansiedad asociados al COVID-19. Interacciones. 2020;6(3):e163.

5. Caycho-Rodríguez T, Barboza-Palomino M, Ventura-León J, Carbajal-León C, Noé-Grijalva $M$, Gallegos M, et al. Traducción al español y validación de una medida breve de ansiedad por la COVID-19 en estudiantes de ciencias de la salud. Ansiedad y Estrés. 2020;26(2-3):174-180.

\section{Correspondencia:}

\section{Andrea Yuliett Cadillo Poma}

Universidad Privada San Juan Bautista, Ica, Perú. Correo electrónico:

andrea.cadillo@upsjb.edu.pe 